\title{
Slide-Down Prevention for Wheeled Mobile Robots on Slopes
}

\author{
Jesús M. García, Jorge L. Martínez*, \\ Anthony Mandow* and Alfonso García-Cerezo* \\ Universidad Nacional Experimental del Táchira, Laboratorio de Prototipos \\ 5001-San Cristobal, Venezuela \\ jmgarcia@unet.edu.ve \\ *Universidad de Málaga, Andalucía Tech, Dpto. Ingeniería de Sistemas y Automática \\ 29071-Málaga, Spain \\ jlmartinez@uma.es, amandow@uma.es, ajgarcia@uma.es
}

\begin{abstract}
Wheeled mobile robots on inclined terrain can slide down due to loss of traction and gravity. This type of instability, which is different from tip-over, can provoke uncontrolled motion or get the vehicle stuck. This paper proposes slide-down prevention by real-time computation of a straightforward stability margin for a given ground-wheel friction coefficient. This margin is applied to the case study of Lazaro, a hybrid skid-steer mobile robot with caster-leg mechanism that allows tests with four or five wheel contact points. Experimental results for both ADAMS simulations and the actual vehicle demonstrate the effectiveness of the proposed approach.
\end{abstract}

Keywords: Mobile robots, inclined terrain, maneuverability, navigation.

\section{$1 \quad$ Introduction}

Mobile robot navigation on inclined terrains is required in challenging environments typically found in disaster areas (Zhang \& Song, 2014), planetary surfaces (Heverly et al., 2013) or agricultural zones (Cariou et al., 2009). Even if special mechanisms have been proposed to improve mobility on steep slopes, such as leg-track hybrid locomotion (Nagatani et al., 2011), plowing tools for loose soil (Loret De Mola Lemus et al., 2014), or an onboard arm pushed against the ground (Serón et al., 2014), many ground robots rely on wheeled locomotion. These wheeled vehicles include Ackermann steering (Freitas et al., 2013), skid-steer locomotion (Britton et al., 2015), differential drive (Ward \& Iagnemma, 2008) or four driven and steered wheels (Lucet et al., 2015).

The hazards encountered by these vehicles include stability problems such as rollover (Chennuri et al., 2015) and sliding down. In practice, it is difficult for a remote operator to detect these potential hazards (Sakurada et al., 2010), so real-time computation of stability margins for operator warning systems are required (Richier et al, 2011) (Suzuki, et al., 2013). Furthermore, stability margins can be used to feed a motion controller or a path planner (Brunner et al., 2012). 
In particular, sliding down can be defined as the uncontrolled motion of the whole vehicle on a slope due to gravity and lack of friction. Sliding down can alter the vehicle's maneuvering ability (Singh \& Krishna, 2016) or get the robot stuck (Yamauchi et al., 2014). However, this relevant problem has received less attention than traction wheel slippage (Balakrishna \& Ghosal, 1995)(Thueer \& Siegwart, 2010), tip-over prevention (Morales et al., 2013), or vehicle sideslip (Cariou, et al., 2009) (Inotsume et al., 2013).

This paper proposes slide-down prevention for wheeled robots by real-time computation of a straightforward stability margin for a given ground-wheel friction coefficient. This margin is applied to the case study of Lazaro (see Fig. 1), a hybrid skid-steer mobile robot with caster-leg mechanism that allows tests with either four or five wheel contact points. Experimental results include ADAMS simulations and real tests while moving in straight-line motion on inclined urban surfaces.

The rest of the paper is organized as follows. The next section proposes the slidedown margin. Section III discusses the case study of the mobile robot Lazaro. The last section is devoted to conclusions and future work.

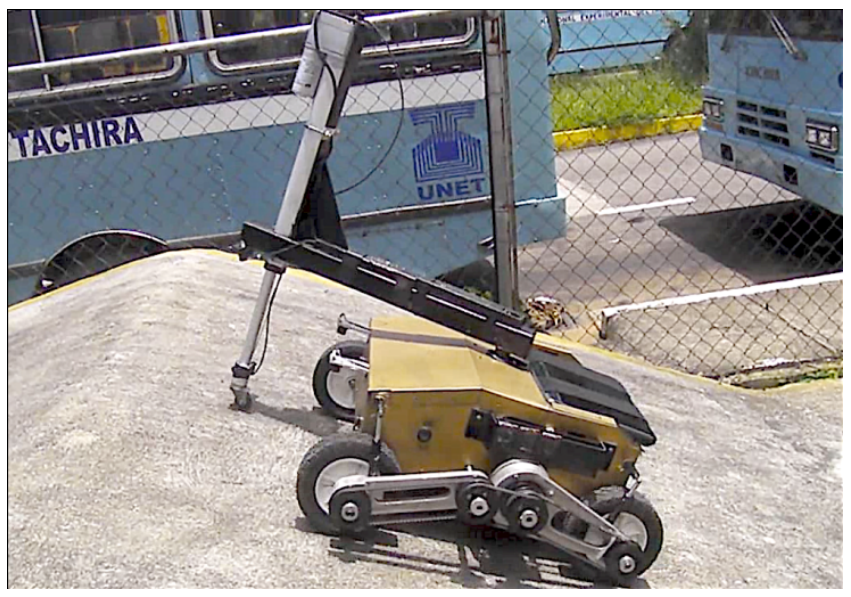

Fig. 1. Lazaro mobile robot on sloped terrain.

\section{Slide-Down Margin}

\subsection{Robot Configuration}

An example of robotic vehicle considered in this paper is shown in Fig. 2. This is a hybrid skid-steer mobile robot with a swivel caster-ended leg mechanism. In this robot, two different configurations can be considered: with or without caster contact.

Let the robot reference frame $X_{c} Y_{c} Z_{c}$ be defined with $X_{c}$ aligned with the forward motion direction of the vehicle and $Z_{c}$ normal to the ground plane and pointing upwards (see Fig. 2). The following assumptions are considered:

- Both the vehicle and onboard mechanisms move at low speeds, so no relevant inertial accelerations apart from gravitation are present. 
- All contact points belong to the same inclined plane, which is reasonable for relatively small-sized robots. The $X_{c} Y_{c}$ plane is defined to coincide with this inclined plane.

- The direction of adhesion forces $F_{i r}$ provided by the ground to the $i^{\text {th }}$ wheel coincides with the maximum slope of the terrain. For traction wheels (numbered one to four in Fig. 2) it will be upwards regardless of vehicle moving direction. For swivel caster wheels (number five in Fig. 2), the adhesion forces will be negligible because the friction with the ground only serves to roll the wheels.

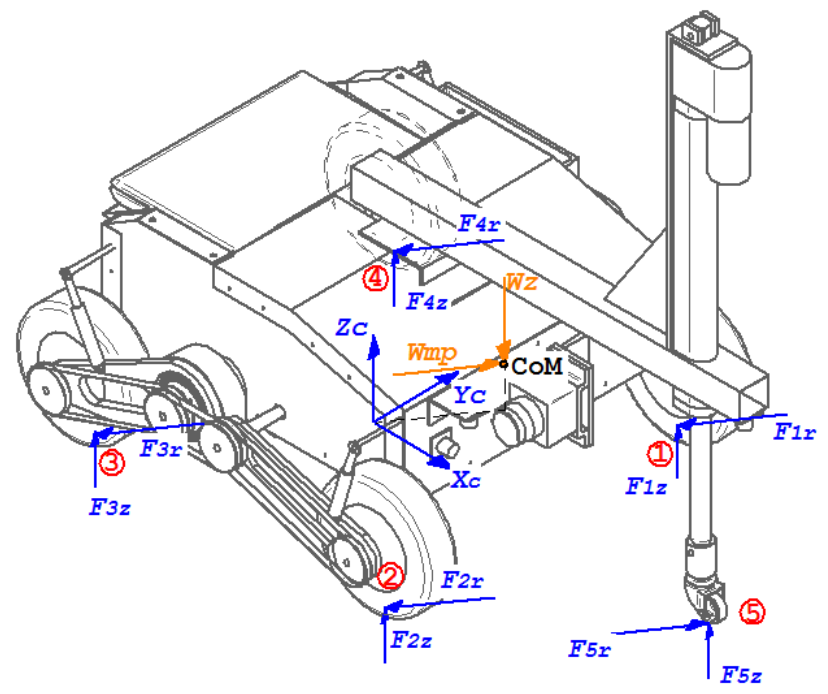

Fig. 2. Adhesion forces diagram for a four-wheeled vehicle with an additional caster wheel. Contact points are numbered one to five.

\subsection{Geometric Approach}

When there are no caster wheels in contact with the ground, the following geometric equation can be employed as a slide-down indicator:

$$
I_{s}^{*}=1-\frac{\tan (\theta)}{\mu}
$$

where $\mu$ is the static friction coefficient and $\theta$ represents the inclination of $X_{c} Y_{c}$ with respect to the horizontal plane:

$$
\theta=\arctan \left(\frac{\sqrt{\sin (\phi)^{2}+\cos (\phi)^{2} \sin (\alpha)^{2}}}{\cos (\phi) \cos (\alpha)}\right),
$$


where $\alpha$ and $\phi$ are the roll and pitch angles of the vehicle, respectively. Combining (1) with (2) gives:

$$
I_{s}^{*}=1-\frac{\sqrt{\sin (\phi)^{2}+\cos (\phi)^{2} \sin (\alpha)^{2}}}{\mu \cos (\phi) \cos (\alpha)} .
$$

This index varies from one to zero, where values close to zero indicate the maximum slide-down risk and values near one represent the minimum on horizontal plane. To compute $I_{s}^{*}$, it is necessary to know $\mu$ beforehand and to obtain the actual pitch and roll angles of the vehicle.

\subsection{Force Balance}

When there are caster wheels in contact with the ground, the above geometric approach fails, and it is necessary to employ a force analysis. For traction wheels, according to Coulomb's friction law, the maximum magnitude of $F_{i r}$ without slippage is:

$$
F_{i r} \leq \mu F_{i z},
$$

where $F_{i z}$ is the normal force of the wheel on the inclined plane (Thibodeau et al., 2006) (Nakamura et al., 2007).

Let $W_{m p}$ be the projection of the weight vector from the Center of Mass (CoM) on the $X_{c} Y_{c}$ plane, whose magnitude is:

$$
W_{m p}=W \sqrt{\sin (\phi)^{2}+\cos (\phi)^{2} \sin (\alpha)^{2}}
$$

where $W$ is the weight of the vehicle. Then, Newton's second law provides the following relationships for the direction of the maximum slope, contained in the $X_{c} Y_{c}$ plane:

$$
\sum_{\forall i} F_{i r}=W_{m p}
$$

and for the $Z_{c}$ direction:

$$
\sum_{\forall i} F_{i z}=W_{z}-\sum_{\forall j} F_{j z}
$$

where indices $i$ and $j$ refer to traction and caster wheels, respectively, and $W_{z}$ is the magnitude of the weight vector normal to the $X_{c} Y_{c}$ plane: 


$$
W_{z}=W \cos (\phi) \cos (\alpha) \text {. }
$$

Vehicle slide-down is avoided as long as (4) applies for all traction wheels, which implies:

$$
\sum_{\forall i} F_{i r} \leq \mu \sum_{\forall i} F_{i z}
$$

Then, using (6) and (7) in (9), the following inequality needs to be fulfilled to avoid slide-down:

$$
W_{m p} \leq \mu\left(W_{z}-\sum_{\forall j} F_{j z}\right),
$$

and the following vehicle slide-down margin can be formulated:

$$
I_{s}=\frac{\mu\left|W_{z}-\sum_{\forall j} F_{j z}\right|-\left|W_{m p}\right|}{\mu\left|W_{z}-\sum_{\forall j} F_{j z}\right|} .
$$

Apart from knowing $\mu, \alpha$ and $\phi$, computation of $I_{s}$ requires to estimate or measure every $F_{j z}$ for caster wheels.

\section{Case Study}

\subsection{The Lazaro Mobile Robot}

Lazaro (see Fig. 1) is a small battery-operated mobile robot with four skid-steer traction wheels that has been specially designed to have an additional contact point with the ground via its onboard leg (García et al., 2015). This leg is a two-degree-offreedom mechanism whose end-effector is a swivel caster wheel, which can be used to improve stability and to negotiate surface discontinuities.

Lazaro weighs $W=255.1 \mathrm{~N}$, of which $36.9 \mathrm{~N}$ correspond to the leg. The dimensions of the vehicle are $425 \mathrm{~mm}$ (width), $468 \mathrm{~mm}$ (length) and $252 \mathrm{~mm}$ (height). The left and right side motors have angular encoders to compute approximate odometry (Mandow et al., 2007).

The $\theta_{1}$ angle of the first revolute joint of the caster wheel is measured by an encoder. This joint allows unlimited rotation of the leg. The second prismatic joint of the mechanism allows a vertical displacement $d_{2}$, which is obtained with a potentiometer. These values can be considered to compute modifications in the CoM position due to leg motion.

To calculate $I_{s}$, the roll and pitch angles with respect to the horizontal plane are measured by an onboard Inertial Measurement Unit (IMU). The force that the linear 
actuator of the second joint exerts against the ground (i.e., $F_{5 z}$ ) is measured by a force sensor over the caster wheel. Finally, the value of $\mu$ can be known by considering that traction wheels are solid rubber, and taking into account the terrain characteristics.

\subsection{ADAMS simulations}

An accurate 3D model of Lazaro was developed with the Solid Edge software. Then, this model was exported to the ADAMS environment (see Fig. 3), where physical properties were assigned to each body of the robot and the relationships between these bodies were defined.

Simulations have been performed on a slippery test surface designed so that the roll angle increases progressively as the vehicle moves in a straight line starting from a flat region (see Fig. 3). Concretely, the friction coefficient between the traction wheels and the test surface was defined as $\mu=0.5$. In the experiments, Lazaro moved at a speed of $0.065 \mathrm{~m} / \mathrm{s}$ with the leg aligned forwards with the vehicle's longitudinal axis.

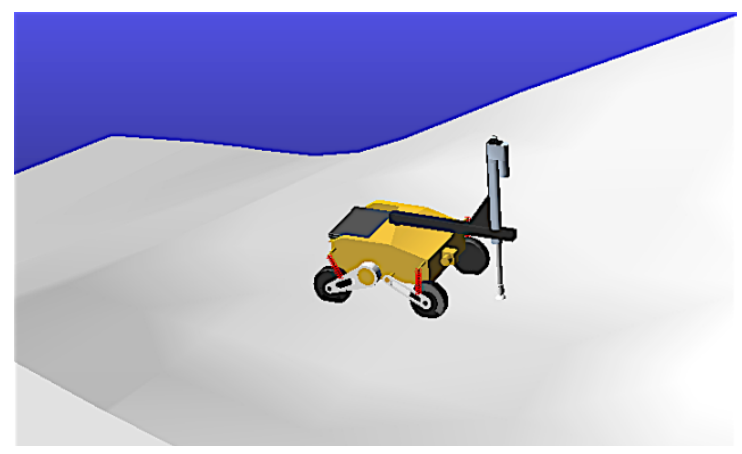

Fig. 3. ADAMS model of Lazaro over a test surface.

In the simulation, the actual adhesion forces of the traction wheels are given by ADAMS, which allows (9) to be evaluated directly. Thus, the vehicle slide-down margin can be computed alternatively as:

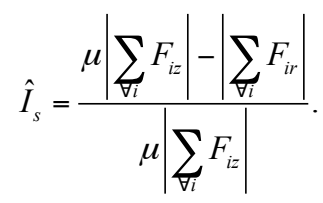

Fig. 4 shows the slide-down margins computed with (11) and (12), the lateral velocity of the robot $v_{y}$, and the roll angle for four different values of $F_{5 z}$, one of which $\left(F_{5 z}=0 \mathrm{~N}\right)$ corresponds to a four-wheeled vehicle configuration. It can be observed that the estimations of the vehicle slide-down margin given by (11) and (12) are very similar, being the mean error between both estimations less than 0.08 (i.e., 
$8 \%$ ). The fluctuations in Fig. 4(b) are because one traction wheel loses and gains contact with the curved surface, especially with $F_{5 z}=72.5 \mathrm{~N}$.

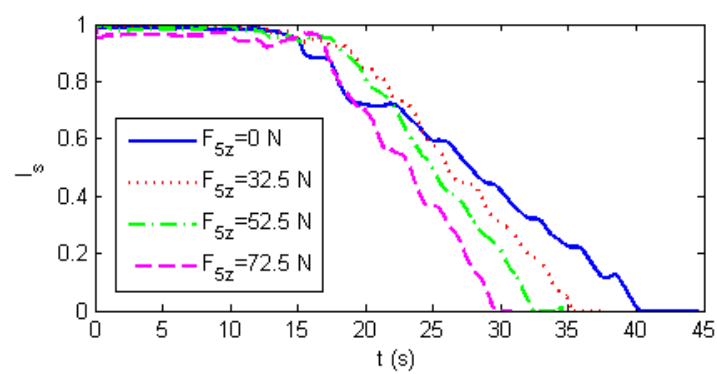

a)

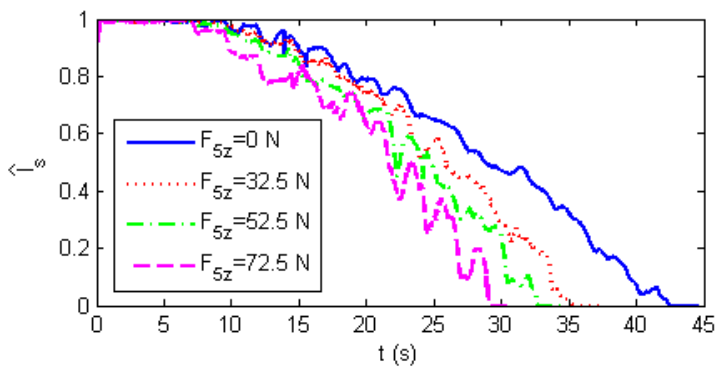

b)

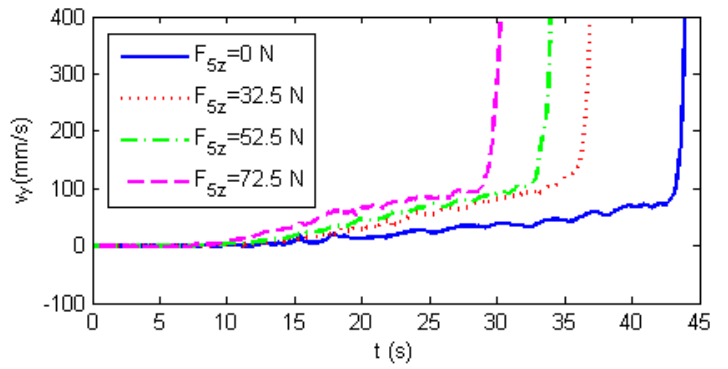

c)

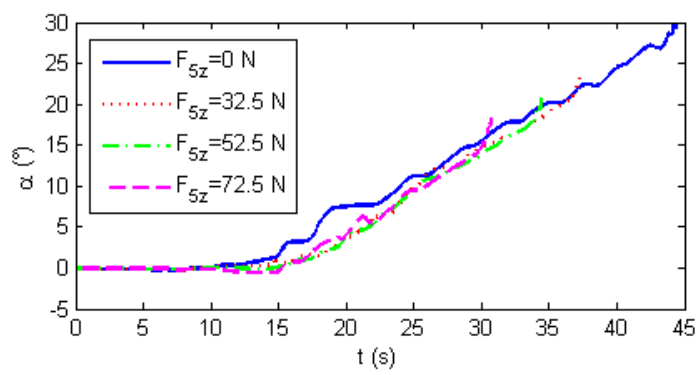

d)

Fig. 4. ADAMS experiments for different caster contact forces: Slide-down margins calculated with (11) (a) and (12) (b); lateral vehicle speed (c); and roll angle (d). 
Three different phases can be identified in Fig. 4(c) as $\alpha$ increases. First, up to approximately $10 \mathrm{~s}$, the vehicle moves on a leveled surface so no lateral velocities appear and $I_{s} \approx 1$. Second, the vehicle goes on moving forward but sideslip appears (i.e., $v_{y}>0$ ). In this phase, as $\alpha$ grows it can be observed that sideslip increases and $I_{s}$ decreases from one to zero. The third phase is characterized by a breakpoint in lateral velocity, where forward motion is interrupted and uncontrolled slide-down occurs. This situation is correctly predicted by $I_{S}$ values close to zero.

As for the effect of the additional contact point, it can be noticed in Fig. 4 the relevant influence of $F_{5 z}$ on sliding as indicated by (10) because greater forces implies smaller $\alpha$ angles to reach the breakpoint.

\subsection{Experimental Results}

Experiments with the actual robot were performed on the inner smooth concrete surface of a half-pipe (see Figs. 5-6). This surface has $\mu \approx 0.65$ for solid rubber wheels. In the two tests discussed below the vehicle moved in straight-line motion at a speed of $0.17 \mathrm{~m} / \mathrm{s}$ with the leg aligned with the longitudinal axis.

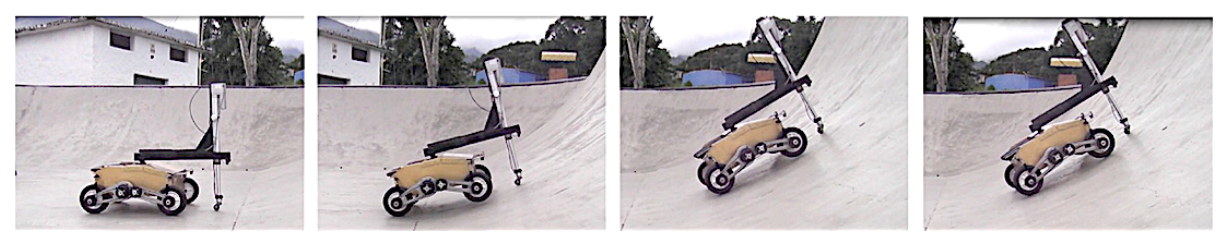

Fig. 5. First test: Image sequence of Lazaro moving upwards on a half-pipe with $\alpha \approx 0^{\circ}$.
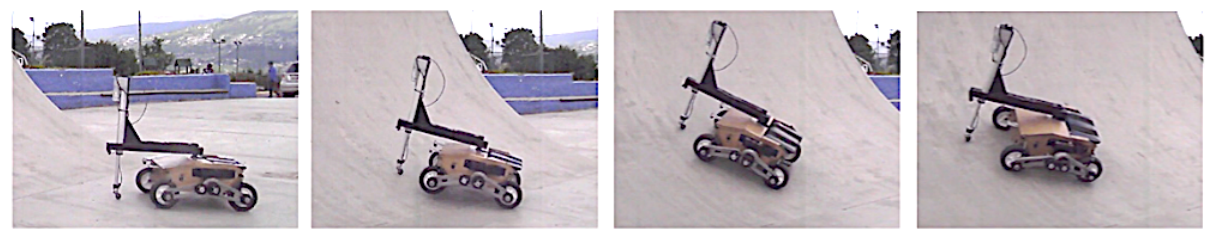

Fig. 6. Second test: Image sequence of Lazaro moving upwards on a half-pipe with varying roll and pitch angles.

The first test illustrates a case dominated by longitudinal slide-down (see Fig. 5). In this test, Lazaro tries to move upwards with $\alpha \approx 0^{\circ}$. The geometric and force-based slide-down margins, the pitch angle $\phi$, and $F_{5 z}$ are shown in Fig. 7. It can be observed that $I_{s}$ decreases as $\phi$ increases. In this case, when the breakpoint is reached, forward motion stops and the vehicle gets stuck. This actually happens when $I_{s}$ approaches zero. The breakpoint phenomenon occurs with $\phi \approx-32^{\circ}$ without additional contact point, whereas for $F_{5 z} \approx 75 \mathrm{~N}$ it occurs with a lower value of $\phi \approx-28^{\circ}$. Regarding $I_{s}^{*}$, it coincides with $I_{s}$ when $F_{5 z}=0 \mathrm{~N}$, but it underestimates the slide-down risk when the caster wheel contacts the ground. 

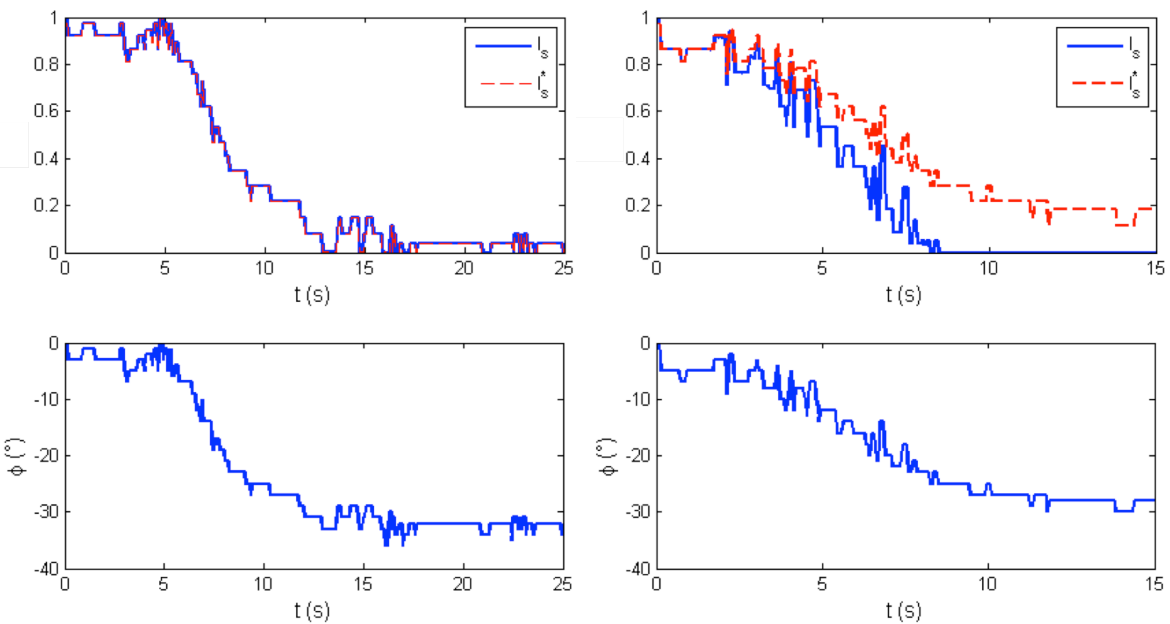

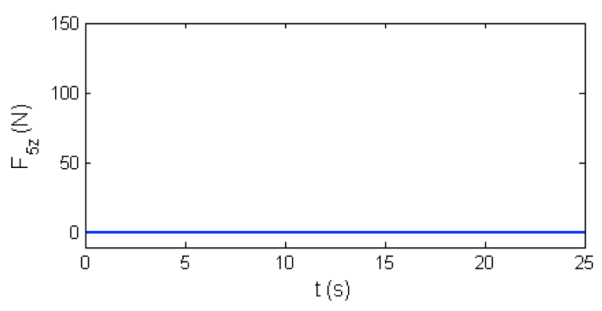

a)

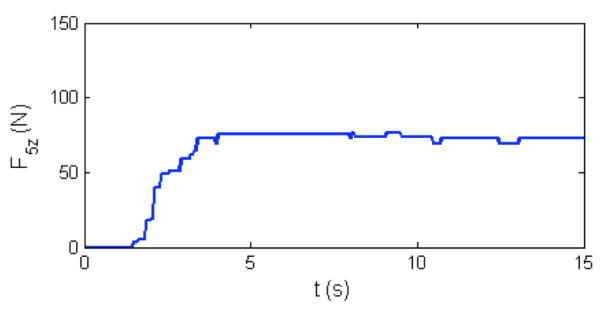

b)

Fig. 7. First test: Slide-down margins calculated with (3) and (11) (up), pitch angle (middle) and applied force (down) with (b) and without (a) additional contact point.

The second test offers a case with lateral slide-down (see Fig. 6). In this test Lazaro moves on a slope of the half-pipe in diagonal without caster contact. The vehicle's pitch and roll angles, and $I_{s}$ during this experiment are shown in Fig. 8. It can be observed that, around $16 \mathrm{~s}$ when $I_{s}$ approaches zero, both $\alpha$ and $\phi$ remain almost unchanged, which means that the vehicle has got stuck. Finally, around $22 \mathrm{~s}$ Lazaro loses its position and orientation completely (see Fig. 6).

\section{Conclusions and Future Work}

Slide-down is a relevant problem for ground robots when moving on steep slopes because it can alter significantly the vehicle position and orientation or get the vehicle stuck. This paper has addressed slide-down prevention for wheeled robots by proposing an easy to compute stability margin for a given ground-wheel friction coefficient based on measured roll and pitch angles and the estimation or measurement of casters normal forces. The proposed margin could be applied for remote operation warnings as well as for motion control and path planning. 

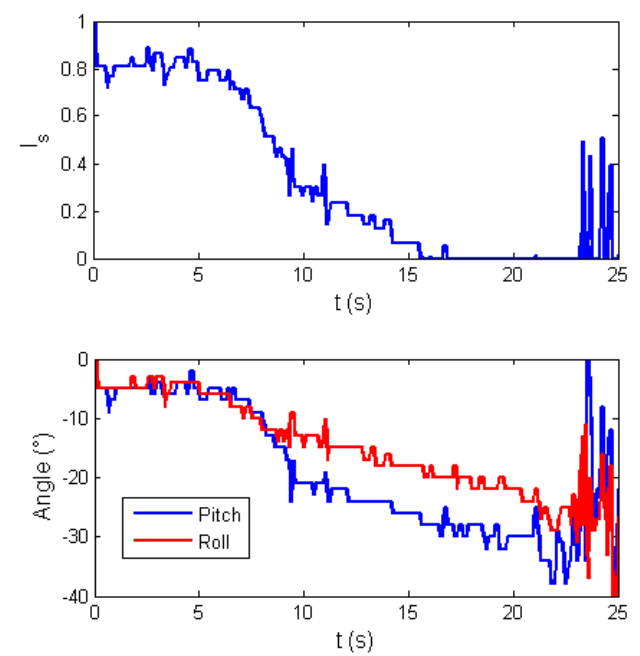

Fig. 8. Second test: Slide-down margin (up), and roll and pitch angles (down) during movement with variable roll and pitch angles.

This margin has been illustrated with the case study of Lazaro, a hybrid skid-steer mobile robot with caster-leg mechanism. This mechanism has allowed testing with both four wheels and five wheels contact. Experimental results for both ADAMS simulations and the actual vehicle on inclined urban surfaces with progressively increasing slope have demonstrated the effectiveness of the proposed approach. Simulations have indicated that the straightforward computation of the proposed margin is consistent with realistic multi-body dynamics performance. Furthermore, real experiments have shown an appropriate decrease to zero of the computed margin value as the vehicle approached slide-down instability.

Future work includes the online estimation of the soil-wheel friction coefficient with onboard inertial measurement. It is also necessary to study the influence of turning with skid-steering and of non-swivel passive rolling wheels on slide-down.

\section{Acknowledgments}

This work was partially supported by Decanato de Investigación de la Universidad Nacional Experimental del Táchira through project 01-020-2010, Spanish project DPI 2015-65186-R, Andalusian project PE-2010 TEP-6101, and Asociación Universitaria Iberoamericana de Posgrado (AUIP).

\section{References}

Balakrishna, R. \& Ghosal, A. (1995). Modeling of Slip For Wheeled Mobile Robots. IEEE Transactions on Robotics and Automation, 11 (1), pp. 126-132. 
Britton, N., Yoshida, K., Walker, J., Nagatani, K., Taylor, G. \& Dauphin, L. (2015). Lunar micro rover design for exploration through virtual reality tele-operation. Springer Tracts in Advanced Robotics, 105, 259-272.

Brunner, M., Brüggemann, B. \& Schulz, D. (2012). Autonomously traversing obstacles: Metrics for path planning of reconfigurable robots on rough terrain. Proc. International Conference on Informatics in Control, Automation and Robotics, (pp. 1-10). Rome, Italy.

Cariou, C., Lenain, R., Thuilot, B. \& Berducat, M. (2009). Automatic guidance of a four-wheel-steering mobile robot for accurate field operations. Journal of Field Robotics, 26 (6-7), 504-518.

Chennuri, V., Kothagadi, H. \& Mohammad, R. (2015). Design and stress analysis of four-post rollover protective structure of agricultural-wheeled tractor. International Journal of Mechanical Engineering and Robotics Research, 4 (1), 292-305.

Freitas, G., Lizarralde, F., Hsu, L. \& Bergerman, M. (2013). Terrain model-based anticipative control for articulated vehicles with low bandwidth actuators. Proc. IEEE International Conference on Robotics and Automation, (pp. 382389). Karlsruhe, Germany.

García, J. M., Martínez, J. L., Mandow, A. \& García-Cerezo, A. (2015). Steerability analysis on slopes of a mobile robot with a ground contact arm. Proc. 23rd Mediterranean Conference on Control and Automation, (pp. 267-272). Torremolinos, Spain.

Heverly, M., Matthews, J., Lin, J., Fuller, D., Maimone, M., Biesiadecki, J. \& Leichty, J. (2013). Traverse performance characterization for the mars science laboratory rover. Journal of Field Robotics, 30 (6), 835-846.

Inotsume, H., Sutoh, M., Nagaoka, K., Nagatani, K. \& Yoshida, K. (2013). Modeling, analysis, and control of an actively reconfigurable planetary rover for traversing slopes covered with loose soil. Journal of Field Robotics, 30 (6), 875-896.

Loret De Mola Lemus, D., Kohanbash, D., Moreland, S. \& Wettergreen, D. (2014). Slope descent using plowing to minimize slip for planetary rovers. Journal of Field Robotics, 31 (5), 770-786.

Lucet, E., Lanain, R. \& Grand, C. (2015). Dynamic path tracking control of a vehicle on slippery terrain. Control Engineering Practice, 42, 60-73.

Mandow, A., Martínez, J. L., Morales, J., Blanco, J. L., García-Cerezo, A. \& González, J. (2007). Experimental kinematics for wheeled skid-steer mobile robots. Proc. IEEE/RSJ International Conference on Intelligent Robots and Systems, (pp. 1222-1227). San Diego, USA.

Morales, J., Martínez, J. L., Mandow, A., Serón, J. \& García-Cerezo, A. (2013). Static tip-over stability analysis for a robotic vehicle with a single-axle trailer on slopes based on altered supporting polygons. IEEE/ASME Transactions on Mechatronics, 18 (2), 697-705.

Nagatani, K., Kinoshita, H., Yoshida, K., Tadakuma, K. \& Koyanagi, E. (2011). Development of leg-track hybrid locomotion to traverse loose slopes and irregular terrain. Journal of Field Robotics, 28 (6), 950-960. 
Nakamura, S., Faragalli, M., Mizukami, N., Nakatani, I., Kunii, Y. \& Kubota, T. (2007). Wheeled robot with movable center of mass for traversing over rough terrain. Proc. IEEE/RSJ International Conference on Intelligents Robots and Systems, (pp. 1228-1233). San Diego, USA.

Richier, M., Lenain, R., Thuilot, B. \& Debain, C. (2011). On-line estimation of a stability metric including grip conditions and slope: Application to rollover prevention for all-terrain vehicles. Proc. IEEE International Conference on Intelligent Robots and Systems, (pp. 4569-4572). San Francisco, USA.

Sakurada, K., Suzuki, S., Ohno, K., Takeuchi, E., Tadokoro, S., Hata, A., Miyahara, N. \& Higashi, K. (2010). Real-time prediction of fall and collision of tracked vehicle for remote-control support. Proc. IEEE/SICE International Symposium on System Integration, (pp. 37-42). Sendai, Japan.

Serón, J., Martínez, J. L., Mandow, A., Reina, A. J., Morales, J. \& García-Cerezo, A. (2014). Automation of the arm-aided climbing maneuver for tracked mobile manipulators. IEEE Transactions on Industrial Electronics, 61 (7), 36383647.

Singh, A. K. \& Krishna, K. M. (2016). Feasible acceleration count: A novel dynamic stability metric and its use in incremental motion planning on uneven terrain. Robotics and Autonomous Systems, 79, 156-171.

Suzuki, S., Hasegawa, S. \& Okugawa, M. (2013). Warning system for crawler-type mobile robot with passive sub-crawler. Proc. IEEE International Symposium on Safety, Security, and Rescue Robotics, (pp. 1-6). Linkoping, Sweden.

Thibodeau, B., Deegan, P. \& Grupen, R. (2006). Static analysis of contact forces with a mobile manipulator. Proc. IEEE International Conference on Robotics and Automation, (pp. 4007-4012). Orlando, USA.

Thueer, T. \& Siegwart, R. (2010). Mobility evaluation of wheeled all-terrain robots. Robotics and Autonomous Systems, 58 (5), 508-519.

Ward, C. C. \& Iagnemma, K. (2008). A dynamic-model-based wheel slip detector for mobile robots on outdoor terrain. IEEE Transactions on Robotics, 24 (4), 821831.

Yamauchi, G., Noyori, T., Nagatani, K. \& Yoshida, K. (2014). Improvement of slope traversability for a multi-DOF tracked vehicle with active reconfiguration of its joint forms. Proc. IEEE International Symposium on Safety, Security and Rescue Robotics, (pp. 1-6). Toyako-cho, Japan.

Zhang, H. \& Song, A. (2014). System centroid position based tipover stability enhancement method for a tracked search and rescue robot. Advanced Robotics, 28 (23), 1571-1585. 